\title{
Effect of Dehydroepiandrosterone on Atherosclerosis in Apolipo- protein E-Deficient Mice
}

\author{
Tadashi Yamakawa $^{1}$, Kikumi Ogihara ${ }^{2}$, Misa Nakamura ${ }^{3}$, Hirotoshi Utsunomiya ${ }^{4}$, Kazuaki Kadonosono ${ }^{5}$, \\ Seigo Kishikawa ${ }^{2}$, and Yasuo Terauchi ${ }^{6}$ \\ ${ }^{1}$ Department of Endocrinology and Diabetes, Yokohama City University Medical Center, Yokohama, Japan \\ ${ }^{2}$ Department of Pathology, College of Environmental Health, Azabu University, Sagamihara, Japan \\ ${ }^{3}$ Department of Physical Therapy, Faculty of Rehabilitation, School of Rehabilitation, Osaka Kawasaki Rehabilitation University, \\ Osaka, Japan \\ ${ }^{4}$ The Department of Pathology, Wakayama Medical University, Wakayama, Japan \\ ${ }^{5}$ Department of Ophthalmology, Yokohama City University Medical Center, Yokohama, Japan \\ ${ }^{6}$ Department of Endocrinology and Metabolism, Yokohama City University School of Medicine, Yokohama, Japan
}

\begin{abstract}
Aim: Several clinical trials have indicated that dehydroepiandrosterone (DHEA) reduces coronary events associated with atherosclerosis. The aim of this study was to examine the inhibitory effect of DHEA on atherosclerosis and the mechanisms involved.

Methods: Apolipoprotein E-knockout (apoE-KO) mice were fed an atherogenic high-cholesterol diet with or without $0.4 \%$ (w/w) DHEA for 12 weeks.

Results: Although the plasma cholesterol and triglyceride levels were not decreased by DHEA, atherosclerotic lesions in the aortic sinus showed a $45 \%$ reduction in area with DHEA treatment versus untreated mice $\left(0.19 \pm 0.01\right.$ vs. $\left.0.10 \pm 0.02 \mu \mathrm{m}^{2} ; p<0.05\right)$.

Accumulation of macrophages in aortic lesions was also markedly reduced in the DHEA group, and the macrophage-positive area decreased to $0.33 \pm 0.06 \mu \mathrm{m}^{2}$ from $0.67 \pm 0.07 \mu \mathrm{m}^{2}(p<0.01)$. Furthermore, DHEA suppressed the expression of monocyte chemoattractant protein-1 in the vessel wall. Thus, inhibition of macrophage infiltration by DHEA reduced the formation of atherosclerotic lesions in apoE-KO mice.
\end{abstract}

Conclusions: DHEA might be an effective agent for clinical management of atherosclerosis, but a larger controlled trial is necessary for confirmation.

JAtheroscler Thromb, 2009; 16:501-508.

Key words; Atherosclerosis, Dehydroepiandrosterone, Apolipoportein E-knockout mice, Monocyte chemoattractant protein-1

\section{Introduction}

Atherosclerosis is a complex vascular disease initiated by abnormal subendothelial accumulation of plasma lipoproteins in the arteries ${ }^{1)}$. Macrophages within the vascular wall ingest cholesterol, resulting in

Address for correspondence: Tadashi Yamakawa, Department of Endocrinology and Diabetes, Yokohama City University Medical Center, 4-57 Urafunecho, Minamiku, Yokohama 2320024, Japan

E-mail: yamakat@urahp.yokohama-cu.ac.jp

Received: July 31, 2008

Accepted for publication: March 13, 2009 transformation into foam cells. Concomitant activation of other cells leads to a chronic inflammatory response in the vessel walls ${ }^{2)}$.

Dehydroepiandrosterone (DHEA) and its sulfate (DHEA-S) are weak but abundant adrenal androgens, although their levels progressively decline with age in both men and women beginning in the third decade $^{3,4)}$. A growing body of opinion suggests that DHEA supplementation may be physiologically beneficial to the elderly in a variety of ways, including prevention of cardiovascular disease ${ }^{5)}$. Various studies concerning the links between DHEA and cardiovascular disease have produced inconsistent results, generat- 
ing much debate and controversy ${ }^{6,7)}$. Prospective population studies of DHEA-S and mortality in men have shown that elevated DHEA-S levels are associated with a lower mortality rate, both due to cardiovascular disease and other causes ${ }^{8}$. Several other longitudinal, cross-sectional, and retrospective studies have examined the relationship between DHEA-S and various aspects of cardiovascular disease. In both men and women, elevated DHEA-S levels were associated with retarded progression of atherosclerosis on coronary angiography ${ }^{9}$. Also, low DHEA-S levels were linked to a higher risk of CVD in some studies ${ }^{10,11)}$, but not in others ${ }^{12,13)}$.

Apolipoprotein E (apoE)-deficient mice show a spontaneous increase of total plasma cholesterol and triglycerides as well as decreased high-density lipoprotein. The atherosclerotic lesions developing in apoEdeficient mice have been found to display a similar morphology to those in humans ${ }^{14)}$; therefore, we investigated the effect of DHEA on atherosclerosis in apoEdeficient mice.

Various adhesion molecules, growth factors, cytokines, and chemoattractants have been implicated in the atherosclerotic process ${ }^{15)}$. One chemoattractant that seems to have a role in early atherogenesis is monocyte chemoattractant protein (MCP)-1, which is strongly expressed in the macrophage-rich regions of human and rabbit atherosclerotic lesions ${ }^{16,17}$. Therefore, to investigate the influence of DHEA on atherogenesis in vivo, apoE-deficient mice were treated with DHEA, and the lesion surface area in the aortic sinus was measured and MCP-1 mRNA expression was assessed.

\section{Materials and Methods}

\section{Mice}

ApoE (-/-) mice were obtained from Jackson Animal Laboratory (Bar Harbor, ME). To create atherosclerosis, mice were placed on the following diet for the indicated period.

The animal care and procedures of the experiments were approved by the animal care committee of Yokohama City University.

\section{Diet}

In the first experiment, 8-week-old apoE-deficient male mice were given a high fat diet containing $20 \%(\mathrm{~g} / 100 \mathrm{~g})$ fat and $0.2 \%$ cholesterol for 2 weeks before the treatment period. Mice were then divided into two groups ( $n=12$ per group): one group received DHEA added to the diet $(0.4 \% \mathrm{w} / \mathrm{w}$ corresponding to $100 \mathrm{mg} / \mathrm{kg} /$ day) and the other group (control) was maintained on the basal diet. DHEA was mixed into the pelletized diet by Oriental Co., Ltd. The dose tested was the same as in previous studies ${ }^{18,19)}$, and the treatment period was 12 weeks. Animals were housed in a room with a 12-h light/dark cycle and were allowed free access to food and water. At the end of the study, animals were anesthetized by inhalation of ether after a 16-h fast, and blood was collected from the retro-orbital plexus for further analysis.

\section{Histological Assessment of Atherosclerotic Lesions}

Several mice from each group were killed at the age of 22 weeks (after 12 weeks on the atherogenic diet, respectively). The proximal aorta and the part of the heart containing the aortic root were removed, equilibrated in $20 \%$ sucrose, and embedded in OCT compound (Tissue-Tek). Cryosections (10 $\mu \mathrm{m}$ thick) spanning a $550-\mu \mathrm{m}$ region of the aortic root were cut, and then 5 sections (every 10th section, i.e., $100 \mu \mathrm{m}$ apart) from each mouse were stained with Sudan III and hematoxylin ${ }^{20)}$. For quantitative analysis of atherosclerotic lesions, the total lesion area in the $5 \mathrm{sec}$ tions from each mouse was measured with NIH Imaging Software according to modification of the method described by Paigen et al. ${ }^{211}$.

\section{Measurement of Lipoproteins}

If HPLC is performed by an on-line single detection technique combined with a selective enzymatic reaction, this allows the lipid constituents of lipoproteins in the column effluent to be detected and monitored without the need for subsequent analysis of column fractions. The on-line detection technique eliminated laborious and time-consuming procedures associated with fraction collection and achieved a high throughput of samples, while improving analytical precision and detection sensitivity; however, this method requires the separate injection of each enzyme reagent, which is both inefficient and wasteful. Multiple injections may be impossible for small samples, e.g., those from individual mice. Usui et al. ${ }^{22)}$ developed a new dual detection HPLC system for lipoprotein analysis that made it possible to simultaneously measure cholesterol and triglycerides in a single sample, thus reducing the number of analytical runs and tests needed. Therefore, plasma lipoproteins at the age of 22 weeks were analyzed by an on-line dual enzymatic method that allowed simultaneous quantification of cholesterol and triglycerides by HPLC at Skylight Biotech Inc. (Akita, Japan) according to the procedure described by Usui et al. ${ }^{22)}$. In brief, $200 \mu \mathrm{L}$ serum diluted to 1:20 with saline was injected into two TSK gel LipopropakXL columns $(300 \times 7.8 \mathrm{~mm}$; Tosoh $)$ connected in tandem, and the levels of cholesterol and 
triglycerides in lipoproteins separated by size were determined using enzymatic reagents prepared by Kyowa Medex (Tokyo, Japan). Total cholesterol and triglyceride concentrations $(\mathrm{mg} / \mathrm{dL})$ were calculated by comparing the total area under the chromatography curves with calibration standards of known concentrations ${ }^{23)}$.

\section{Immunohistochemistry}

Serial cryosections of the aortic root harvested at $100-\mu \mathrm{m}$ intervals were stained with an anti-mouse monocyte/macrophage monoclonal rat antibody (BM8; Hycult Biotecnology, 1:250 dilution) or an anti-human T-cell polyclonal rabbit antibody (CD3; DAKO; 1:100 dilution), followed by detection with biotinylated secondary antibodies and streptavidinhorseradish peroxidase ${ }^{24)}$. Quantitative analysis of the stained area was performed as described previously, and the percent positive area ((stained area/total atherosclerotic lesion area) $\times 100$ ) was calculated ${ }^{25)}$.

\section{Isolation of RNA and Real-Time PCR Analysis}

Aortic arch tissues at the age of 22 weeks were washed briefly with $1 \times$ PBS before RNA extraction. They were homogenized and total RNA was extracted with Trizol reagent (Gibco-BRL Life Technologies). The quantity and quality of each RNA sample were evaluated by spectrophotometry (Beckman Coulter, DU640B) at 260 and $280 \mathrm{~nm}$. RNA quality was also examined by electrophoresis on $1 \%$ formaldehyde agarose RNA gel. Monocyte chemoattractant protein-1 (MCP-1) gene expression was analyzed by real-time quantitative RT-PCR using the TaqMan system with real-time detection of fluorescence (ABI Prism 7700; Perkin-Elmer Inc.). Expression of the target gene sequence was normalized for the expression of an endogenous control, glyceraldehyde-3-phospatedehydrogenase (GAPDH) (GAPDH TaqMan Control Reagent Kit; ABI Prism 7700, Perkin-Elmer Inc.). Primers and TaqMan probes for MCP-1 and the reference (GAPDH) were constructed using Primer Express (ABI Prism 7700; Perkin-Elmer Inc.). The following forward $(\mathrm{F})$ and reverse $(\mathrm{R})$ primers and TaqMan probes $(\mathrm{P})$ were used for the quantification of MCP-1 and GAPDH mRNA: MCP-1 (F), 5'-GCTGCTACTCATTCACTGGCAA-3'; MCP-1 (R), 5'CTGCTGCTGCTGATTCTCTTGT-3'; MCP-1 (P), 5'-TGATCCCAATGAGTCGGCTGGAGAA-3'; GAPDH (F), 5'-TGTTCTAGAGACAGCCGCATCTT-3'; GAPDH (R), 5'-CACCGACCTTCACCATATTGT-3'; and GAPDH (P), 5'-TTGTGCAGTGCCAGCCTCGTCTCATA-3'
Table 1. Plasma cholesterol and triglyceride levels

\begin{tabular}{lccc}
\hline & Control group & DHEA group & $p$ value \\
\hline Total cholesterol (mg/dL) & $1,119 \pm 236$ & $1,212 \pm 144$ & 0.39 \\
Triglycerides $(\mathrm{mg} / \mathrm{dL})$ & $75 \pm 25$ & $99 \pm 29$ & 0.11 \\
\hline
\end{tabular}

Values are the mean \pm SD for eight mice.

Table 2. Plasma cholesterol profile

\begin{tabular}{lccc}
\hline & Control group & DHEA group & $p$ value \\
\hline Cholesterol (mg/dL) & & & \\
CM & $4.4 \pm 1.6$ & $4.4 \pm 1.0$ & 0.99 \\
VLDL & $856 \pm 242$ & $953 \pm 144$ & 0.35 \\
LDL & $231 \pm 23$ & $228 \pm 16$ & 0.82 \\
HDL & $27 \pm 1.7$ & $26 \pm 4.0$ & 0.54 \\
\hline
\end{tabular}

Values are the mean \pm SD for eight mice. VLDL, Very low density lipoprotein; LDL, low density lipoprotein; CM, chyromicron; HDL, high density lipoprotein

\section{Statistical Analysis}

Data are presented as the mean $\pm \mathrm{SD}$. The unpaired Student's $t$-test was used to assess the significance of differences between 2 groups. For analysis of lesion size, comparisons between groups were performed by the Mann-Whitney $U$ test. In these analyses, $p<0.05$ was considered significant.

\section{Results}

\section{DHEA did not Decrease Plasma Lipid Levels or Alter the Cholesterol Distribution in ApoE-Deficient Mice}

Eight-week-old apoE-deficient mice were fed a Western diet for 8 weeks, starting from 2 weeks before DHEA supplementation $(0.4 \% \mathrm{w} / \mathrm{w}$ in the diet, corresponding to $100 \mathrm{mg} / \mathrm{kg} /$ day). When the effect of DHEA on plasma lipids was examined, neither plasma cholesterol concentration nor missing words altered by DHEA compared with control mice (Table 1).

To assess whether DHEA altered the lipoprotein profile, the distribution of cholesterol in the lipoproteins was analyzed by high-resolution HPLC ${ }^{22)}$. Very low density lipoprotein (VLDL) cholesterol was not decreased by DHEA treatment compared with the level in control animals (953 \pm 144 vs. $856 \pm 242 \mathrm{mg} /$ dL). Similarly, DHEA did not decrease low density lipoprotein (LDL) cholesterol (228 \pm 16 vs. $231 \pm 23$ $\mathrm{mg} / \mathrm{dL}$; Table 2). Also, there were no significant differences in chyromicron (CM) or high density lipoprotein (HDL) cholesterol between DHEA-treated and control mice. 

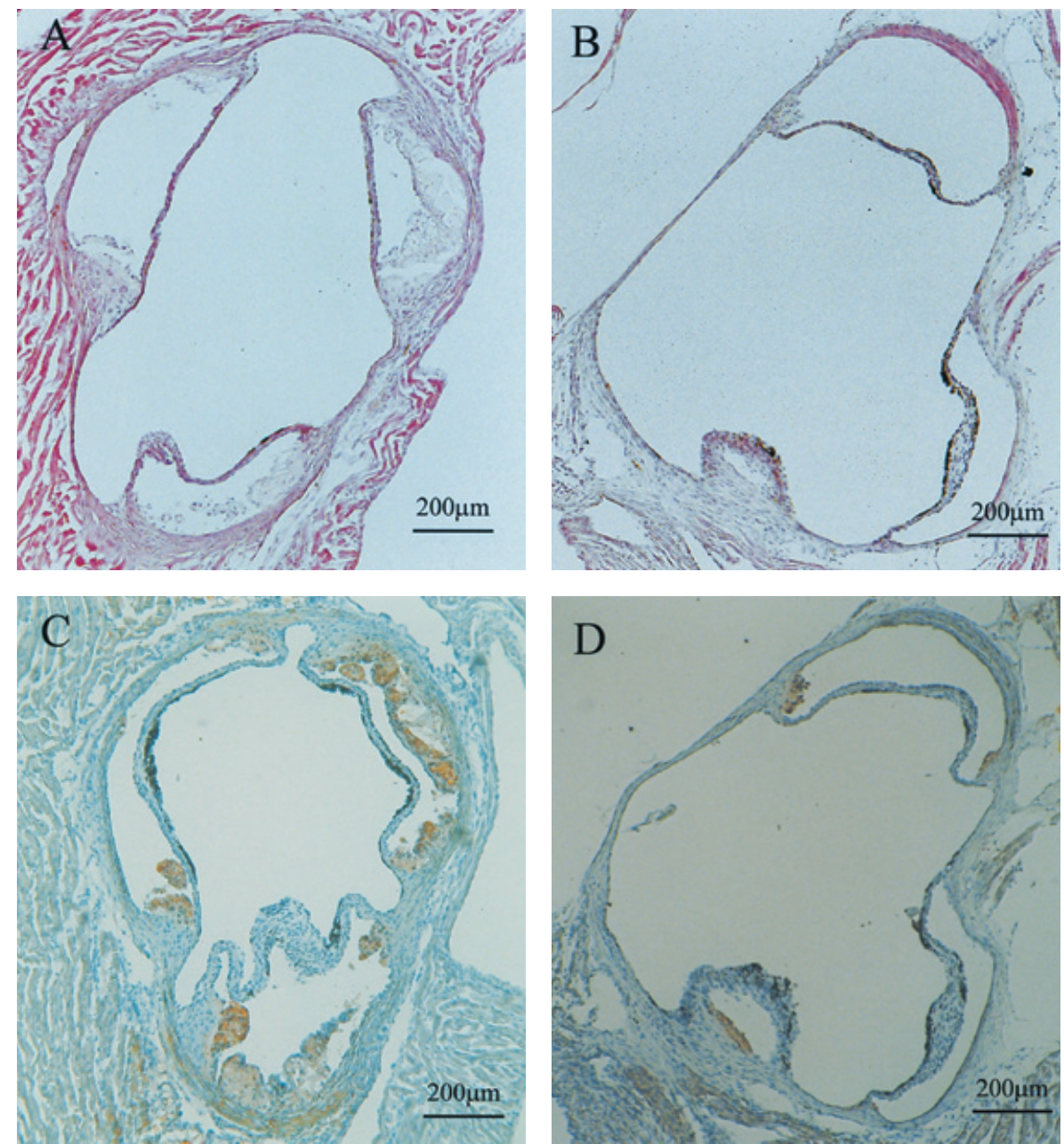

Fig. 1. Light photomicrographs of sections of aortic lesions in apo-E-KO mice maintained on the atherogenic diet for 12 weeks.

A and C, nontreated (control) group. B and D, DHEA-treated (drug) group. Sections were taken at the same level of aortic valves and stained for lipid with hematoxylin and eosin (A and B) for with Sudan III (C and D). Representative images are shown from 8 mice at 22 weeks of age. Original magnification $\times 80$.

\section{DHEA Decreased the Lesion Area in the Aortic Sinus}

The extent of atherosclerotic lesions was measured in serial sections obtained between the aortic valve and the aortic sinus. Representative Sudan IIIstained aortic root sections from apoE-KO mice fed an atherogenic diet are shown in Fig. 1. The crosssectional lesion area was significantly smaller in the DHEA group than in the control group $(45 \% ; 0.10 \pm$ 0.02 vs. $0.19 \pm 0.01 \mu \mathrm{m}^{2}, p<0.05$, Fig. 2 ).

\section{DHEA Reduced Macrophage Infiltration into Ath- erosclerotic Lesions}

Subendothelial macrophages were abundant in the atherosclerotic lesions of control apoE-KO mice fed an atherogenic diet for 8 weeks (Fig. 3A, C), but fewer macrophages were detected in the lesions of DHEA-treated apoE-KO mice (Fig. 3B, D). Quantitative analysis confirmed that the area of BM8-positive staining was significantly reduced in the DHEA group $\left(0.33 \pm 0.06\right.$ vs. $0.67 \pm 0.07 \mu \mathrm{m}^{2}$ per five sections, $p<$ 0.01; Fig. 3E).

\section{Real-Time PCR}

To further assess the mechanisms underlying this reduction of lesions, RNA were isolated from aortae of apo-E-KO mice $(n=8)$ with or without DHEA treatment at 22 weeks of age and MCP-1 mRNA was quantified. DHEA treatment significantly down-regulated the expression of MCP-1 mRNA (2.0 \pm 0.6 vs. $1.0 \pm 0.4 \%, p<0.05 ;$ Fig.4). 


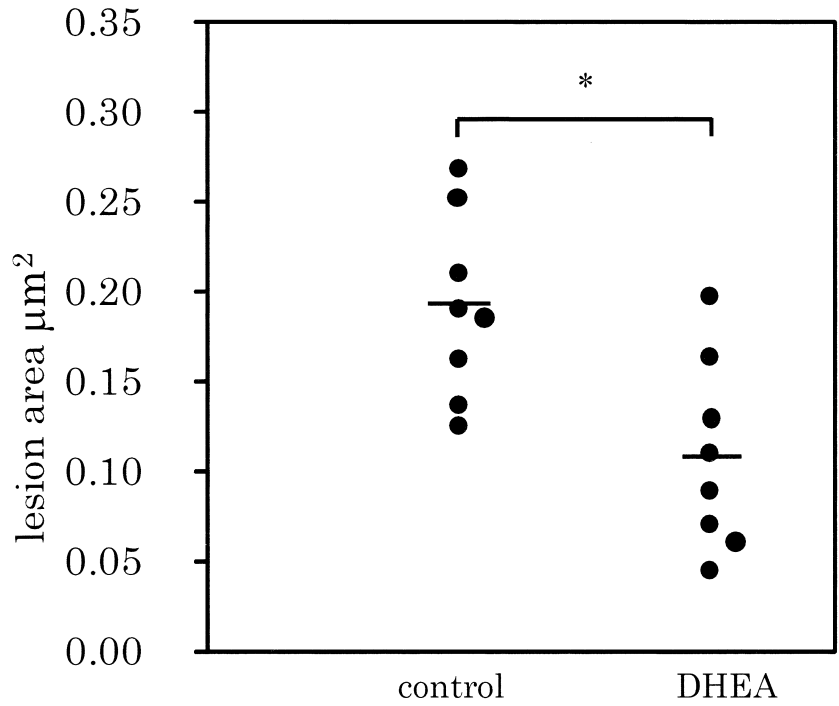

Fig. 2. Dot plot of cross-sectional lesion areas of aortic sinus in apo-E-KO mice maintained on the atherogenic diet for 12 weeks with or without DHEA-treatment.

Aortae were isolated from apo-E-KO mice $(n=8)$ at 22 weeks of age. ${ }^{*} p<0.05$

\section{Discussion}

In the present study, we demonstrated that DHEA treatment could protect apoE-knockout mice against dietary atherosclerosis. We postulated that inhibition of MCP-1 contributed to the suppression of atherosclerosis in these mice, but the possibility remains that other effects on inflammation, cell adhesion, and oxidation also enhanced the antiatherogenic activity of DHEA.

Although the plasma concentration of DHEA is usually considerably higher than that of any other androgen, little information is available concerning its direct actions on the blood vessels ${ }^{26)}$. DHEA has been shown to have an antiatherosclerotic effect in animal models ${ }^{27,28)}$, although the mechanism involved is not yet known. We focused on the effect of DHEA on macrophage infiltration into atherosclerotic lesions, a process that plays a central role in the initiation and progression of atherosclerosis ${ }^{15)}$. No pharmacologic intervention that specifically targets the infiltration of monocytes and/or macrophages is clinically available at present.

DHEA has been shown to reduce the serum level of LDL-cholesterol in asymptomatic humans, although no relationship has been reported between the cholesterol level and the benefits obtained from an increase of plasma $\mathrm{DHEA}^{29)}$. In rabbits, DHEA has been consistently found to decrease atherosclerosis. All stud- ies $^{27,28,30,31)}$ performed in intact or castrated male and female rabbits treated with DHEA for 5 to 30 weeks have shown a significant reduction in the extent of spontaneous or balloon injury-induced aortic or cardiac transplant atherosclerotic lesions, independently of any changes in lipids. These findings were compatible with our results. Thus, the antiatherogenic effects of DHEA are not due to a reduction of serum lipid levels.

The mechanisms by which DHEA exerts its antiatherogenic effect remain unknown. As shown in Fig. 2, macrophage infiltration into atherosclerotic plaques was decreased by treatment with DHEA. In experimental studies, DHEA has been found to promote fibrinolysis ${ }^{32)}$, decrease lipid accumulation in cultured mouse foam cells $s^{33)}$, and limit the proliferation and migration of vascular SMC lines ${ }^{34)}$; therefore, the antiatherosclerotic effect of DHEA might be partly mediated via the suppression of macrophage infiltration.

It is known that several cytokines have a role in atherogenesis. One chemoattractant implicated in the early stages of this process is MCP- ${ }^{16}$, which is strongly expressed in macrophage-rich regions of human and rabbit atherosclerotic lesions ${ }^{25}$. Recently, anti-MCP-1 gene therapy with a deletion mutant of the human MCP-1 gene was reported to inhibit atherosclerosis in apoE-KO mice ${ }^{35)}$. However, few authors have addressed the question of whether DHEA suppresses MCP-1 expression. As shown in Fig. 3, DHEA treatment actually inhibited MCP-1 mRNA expression, an effect that could predominate over stimuli that induce the expression of MCP-1 in macrophagederived foam cells. Quite recently, it was reported that DHEA decreases MCP-1 mRNA expression in endothelial cells ${ }^{36)}$, an observation that matches our findings; however, further studies on the validation of protein for MCP-1 expression are required.

Other mechanisms may also be involved in the antiatherosclerotic effects of DHEA, such as the conversion of DHEA to estrogen, and nitric oxide may play a role in the antiatherosclerotic effects of both DHEA and estrogen ${ }^{31)}$. A higher serum estradiol level is associated with a lower risk of CVD events in older men ${ }^{37)}$. In men, estrogen is mainly synthesized by local tissue aromatization of androgenic precursors released from the testis and adrenal gland, and estrogen levels do not seem to decrease with age to the same extent as those of testosterone or DHEA-S ${ }^{3)}$. To investigate the role of estrogen, it would be interesting to examine whether an aromatase inhibitor could block the antiatherosclerotic effect of DHEA-S in apoE-KO mice and we are now planning to do such experiments.

DHEA has been shown to exert many of its 

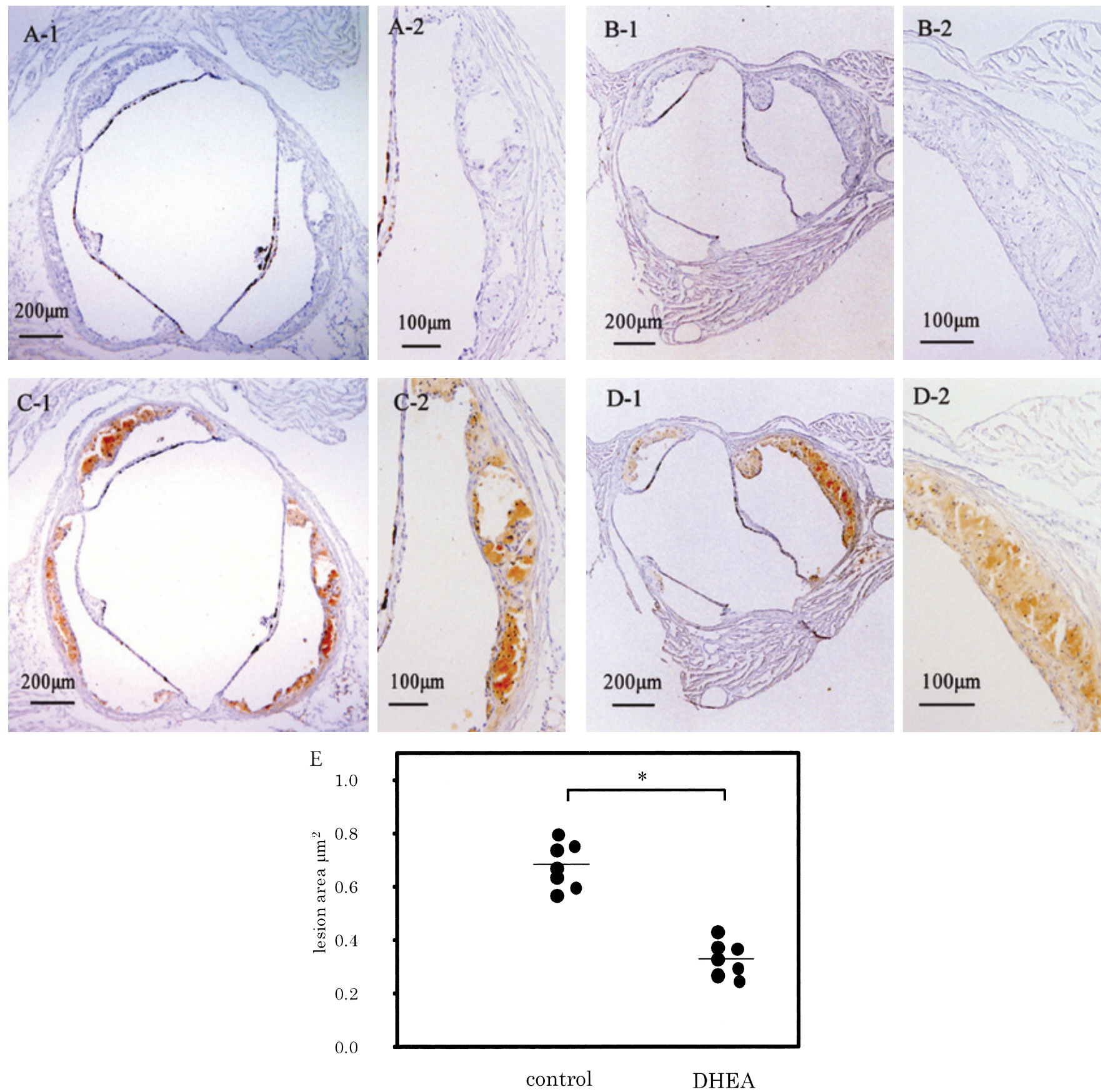

Fig. 3. Photomicrographs of immunostaining for CD3 (A and B) and BM-8 (C and D) of sections of aortic lesions in apo-E-KO mice maintained on the atherogenic diet for 12 weeks.

A and C, nontreated (control) group. B and D, DHEA-treated (drug) group. Representative images are shown from 8 mice at 22 weeks of age. Original magnification $\times 80$ (A-1, B-1, C-1, D-1) and 160 (A-2, B-2, C-2, D-2). E, Dot plot of lesion areas with BM8-positive staining per five sections. ${ }^{*} p<0.05$

effects via the androgen receptor (AR) and/or estrogen receptor (ER) after enzymatic conversion to an androgen or estrogen ${ }^{38}$. In the elderly, the use of DHEA as a dietary supplement is of potential concern in that its androgenic or estrogenic actions may stimulate the proliferation of cancer cells within the prostate or breast and may have other adverse effects. Controversy persists as to whether DHEA enhances or reduces the 


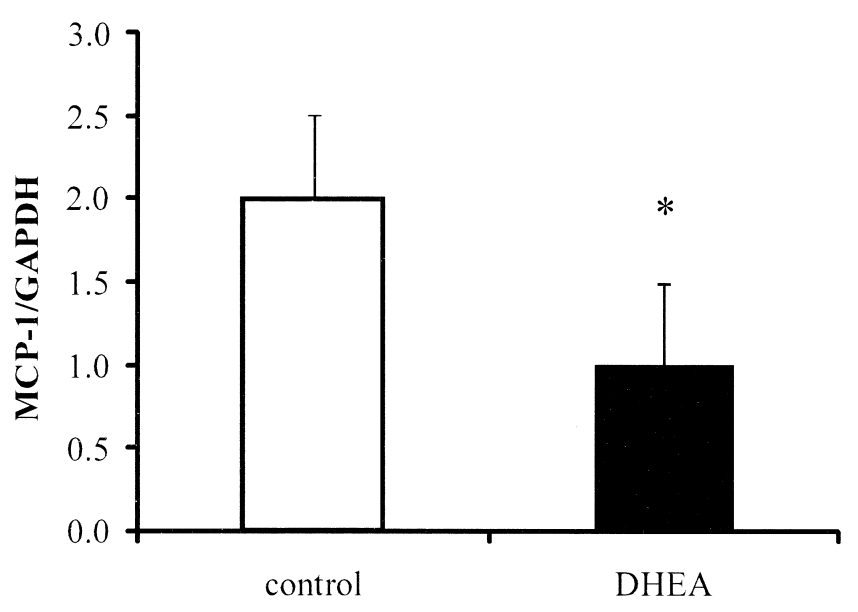

Fig.4. Comparison of MCP-1/GAPDH mRNA expression in apo-E-KO mice maintained on the atherogenic diet for 12 weeks with or without DHEA treatment.

Aortae were isolated from apo-E-KO mice $(n=8)$ at 22 weeks of age. ${ }^{*} p<0.05$

risk of prostate and breast cancer ${ }^{39)}$. Although DHEA and DHEAS have been reported to increase mammary cancer cell proliferation via the $\mathrm{AR}^{40)}$, various other in vivo and in vitro studies conducted with rodents and rodent cells have suggested that DHEA prevents cancer progression ${ }^{41-44)}$. Thus, the precise influence of DHEA on normal and malignant prostate or breast cells remains to be elucidated.

Whether DHEA supplements should be given to replace declining DHEA and DHEA-S levels in aging individuals is a matter of considerable debate ${ }^{6}$. Claims have been made that such treatment could have beneficial effects on a variety of age-related conditions or diseases; however, discrepancies concerning the effects of DHEA in different studies have been a problem, possibly reflecting the small sample size in some instances. In this study, we found that DHEA had a protective effect against atherosclerosis in apoE-KO mice. To resolve the discrepancies regarding the benefits of DHEA, large-scale intervention trials will be necessary.

In conclusion, DHEA was shown to have a protective effect against atherosclerosis in apoE-KO mice, and inhibition of macrophage infiltration was observed after DHEA treatment.

\section{Acknowledgements}

This work was supported by grants-in-aid for Scientific Research on Priority Areas and grants-in-aid for Scientific Research from the Ministry of Education, Science, Sports, and Culture, Japan. We also thank Dr Kenichi Hirata for technical assistance. We are grateful to Mrs. Yuko Watanabe for secretarial assistance and technical support for animal care.

\section{References}

1) Skålén K, Gustafsson M, Rydberg EK, Hultén LM, Wiklund O, Innerarity TL, Borén J: Subendothelial retention of atherogenic lipoproteins in early atherosclerosis. Nature, 2002; 417: 750-754

2) Lusis AJ: Atherosclerosis. Nature, 2000; 407: 233-241

3) Vermeulen A: Dehydroepiandrosterone sulfate and aging. Ann N Y Acad Sci, 1995; 774: 121-127

4) Birkenhäger-Gillesse EG, Derksen J, Lagaay AM: Dehydroepiandrosterone sulphate (DHEAS) in the oldest old, aged 85 and over. Ann N Y Acad Sci, 1994; 719: 543-552

5) Tchernof A, Labrie F: Dehydroepiandrosterone, obesity and cardiovascular disease risk: a review of human studies. Eur J Endocrinol, 2004; 151: 1-14

6) Skolnick AA: Scientific verdict still out on DHEA. JAMA, 1996; 276: 1365-1367

7) Nippoldt TB: Dehydroepiandrosterone supplements: bringing sense to sensational claims. Endocr Pract, 1998; 4: 106-111

8) Barrett-Connor E, Khaw KT, Yen SS: A prospective study of dehydroepiandrosterone sulfate, mortality, and cardiovascular disease. N Engl J Med, 1986; 315: 1519-1524

9) Herrington DM: Dehydroepiandrosterone and coronary atherosclerosis. Ann N Y Acad Sci, 1995; 774: 271-280

10) Trivedi DP, Khaw KT: Dehydroepiandrosterone sulfate and mortality in elderly men and women. J Clin Endocrinol Metab, 2001; 86: 4171-4177

11) Feldman HA, Johannes $C B$, Araujo $A B$, Mohr BA, Longcope $\mathrm{C}$, McKinlay JB: Low dehydroepiandrosterone and ischemic heart disease in middle-aged men: prospective results from the Massachusetts Male Aging Study. Am J Epidemiol, 2001; 153: 79-89

12) Hauner H, Stangl K, Burger K, Busch U, Blömer H, Pfeiffer EF: Sex hormone concentrations in men with angiographically assessed coronary artery disease--relationship to obesity and body fat distribution. Klin Wochenschr, 1991; 69: 664-668

13) Phillips GB, Pinkernell BH, Jing TY: The association of hypotestosteronemia with coronary artery disease in men. Arterioscler Thromb, 1994; 14: 701-706

14) Reddick RL, Zhang SH, Maeda N: Atherosclerosis in mice lacking apo E. Evaluation of lesional development and progression. Arterioscler Thromb, 1994; 14: 141-147

15) Ross R: Cell biology of atherosclerosis. Annu Rev Physiol, 1995; 57: 791-804

16) Nelken NA, Coughlin SR, Gordon D, Wilcox JN: Monocyte chemoattractant protein-1 in human atheromatous plaques. J Clin Invest, 1991; 88: 1121-1127

17) Yet SF, Layne MD, Liu X, Chen YH, Ith B, Sibinga NE, Perrella MA: Absence of heme oxygenase-1 exacerbates atherosclerotic lesion formation and vascular remodeling, FASEB J, 2003; 17: 1759-1761

18) Coleman DL, Leiter EH, Applezweig N: Therapeutic effects of dehydroepiandrosterone metabolites in diabetes mutant mice (C57BL/KsJ-db/db). Endocrinology, 1984; 
115: 239-243

19) Aoki K, Saito T, Satoh S, Mukasa K, Kaneshiro M, Kawasaki S, Okamura A, Sekihara H: Dehydroepiandrosterone suppresses the elevated hepatic glucose-6-phosphatase and fructose-1,6-bisphosphatase activities in C57BL/Ksj$\mathrm{db} / \mathrm{db}$ mice: comparison with troglitazone. Diabetes, 1999; 48: 1579-1585

20) Gosling J, Slaymaker S, Gu L, Tseng S, Zlot CH, Young SG, Rollins BJ, Charo IF: MCP-1 deficiency reduces susceptibility to atherosclerosis in mice that overexpress human apolipoprotein B. J Clin Invest, 1999; 103: 773-778

21) Paigen B, Morrow A, Holmes PA, Mitchell D, Williams RA: Quantitative assessment of atherosclerotic lesions in mice. Atherosclerosis, 1987; 68: 231-240

22) Usui S, Hara Y, Hosaki S, Okazaki M: A new on-line dual enzymatic method for simultaneous quantification of cholesterol and triglycerides in lipoproteins by HPLC. J Lipid Res, 2002; 43: 805-814

23) Okazaki M, Usui S, Hosaki S: In: Rifai N, Warnick GR, and Dominiczak MH (eds) Handbook of Lipoprotein Testing, Second Ed ed. American Association of Clinical Chemistry Press, Washington, D.C., 2000, pp 647-669

24) Boring L, Gosling J, Cleary M, Charo IF: Decreased lesion formation in CCR2-/- mice reveals a role for chemokines in the initiation of atherosclerosis. Nature, 1998; 394: 894-897

25) Ylä-Herttuala S, Lipton BA, Rosenfeld ME, Särkioja T, Yoshimura T, Leonard EJ, Witztum JL, Steinberg D: Expression of monocyte chemoattractant protein 1 in macrophage-rich areas of human and rabbit atherosclerotic lesions. Proc Natl Acad Sci USA, 1991; 88: 5252-5256

26) Okabe T, Haji M, Takayanagi R, Adachi M, Imasaki K, Kurimoto F, Watanabe T, Nawata H: Up-regulation of high-affinity dehydroepiandrosterone binding activity by dehydroepiandrosterone in activated human $\mathrm{T}$ lymphocytes. J Clin Endocrinol Metab, 1995; 80: 2993-2996

27) Gordon GB, Bush DE, Weisman HF: Reduction of atherosclerosis by administration of dehydroepiandrosterone. A study in the hypercholesterolemic New Zealand white rabbit with aortic intimal injury. J Clin Invest, 1988; 82: $712-720$

28) Arad Y, Badimon JJ, Badimon L, Hembree WC, Ginsberg $\mathrm{HN}$ : Dehydroepiandrosterone feeding prevents aortic fatty streak formation and cholesterol accumulation in cholesterol-fed rabbit. Arteriosclerosis, 1989; 9: 159-166

29) Orentreich N, Brind JL, Rizer RL, Vogelman JH: Age changes and sex differences in serum dehydroepiandrosterone sulfate concentrations throughout adulthood. J Clin Endocrinol Metab, 1984; 59: 551-555

30) Eich DM, Nestler JE, Johnson DE, Dworkin, GH, Ko D, Wechsler AS, Hess ML: Inhibition of accelerated coronary atherosclerosis with dehydroepiandrosterone in the heterotopic rabbit model of cardiac transplantation. Circulation, 1993; 87: 261-269

31) Hayashi T, Esaki T, Muto E, Kano H, Asai Y, Thakur NK, Sumi D, Jayachandran M, Iguchi A: Dehydroepiandrosterone retards atherosclerosis formation through its conversion to estrogen: the possible role of nitric oxide. Arterioscler Thromb Vasc Biol, 2000; 20: 782-792

32) Beer NA, Jakubowicz DJ, Matt DW, Beer RM, Nestler
JE: Dehydroepiandrosterone reduces plasma plasminogen activator inhibitor type 1 and tissue plasminogen activator antigen in men. Am J Med Sci, 1996; 311: 205-210

33) Taniguchi S, Yanase T, Kobayashi K, Takayanagi R, Nawata H: Dehydroepiandrosterone markedly inhibits the accumulation of cholesteryl ester in mouse macrophage J774-1 cells. Atherosclerosis, 1996; 126: 143-154

34) Furutama D, Fukui R, Amakawa M, Ohsawa N: Inhibition of migration and proliferation of vascular smooth muscle cells by dehydroepiandrosterone sulfate. Biochim Biophys Acta, 1998; 1406: 107-114

35) Ni W, Egashira K, Kitamoto S, Kataoka C, Koyanagi M, Inoue S, Imaizumi K, Akiyama C, Nishida KI, Takeshita A: New anti-monocyte chemoattractant protein-1 gene therapy attenuates atherosclerosis in apolipoprotein E-knockout mice. Circulation, 2001; 103: 2096-2101

36) Gutiérrez G, Mendoza C, Zapata E, Montiel A, Reyes E, Montaño LF, López-Marure R: Dehydroepiandrosterone inhibits the TNF-alpha-induced inflammatory response in human umbilical vein endothelial cells. Atherosclerosis, 2007; 190: 90-99

37) Arnlöv J, Pencina MJ, Amin S, Nam BH, Benjamin EJ, Murabito JM, Wang TJ, Knapp PE, D'Agostino RB, Sr.Bhasin S, Vasan RS: Endogenous sex hormones and cardiovascular disease incidence in men. Ann Intern Med, 2006; 145: 176-184

38) Labrie F, Bélanger A, Luu-Thev, Labrie C, Simard J, Cusan L, Gomez JL, Candas B: DHEA and the intracrine formation of androgens and estrogens in peripheral target tissues: its role during aging. Steroids, 1998; 63: 322-328

39) Acacio BD, Stanczyk FZ, Mullin P, Saadat P, Jafarian N, Sokol RZ: Pharmacokinetics of dehydroepiandrosterone and its metabolites after long-term daily oral administration to healthy young men. Fertil Steril, 2004; 81: 595-604

40) Bégin D, Luthy IA, Labrie F: Adrenal precursor C19 steroids are potent stimulators of growth of androgen-sensitive mouse mammary carcinoma Shionogi cells in vitro. Mol Cell Endocrinol, 1988; 58: 213-219

41) Green JE, Shibata MA, Shibata E, Moon RC, Anver MR, Kelloff G, Lubet R: 2-Difluoromethylornithine and dehydroepiandrosterone inhibit mammary tumor progression but not mammary or prostate tumor initiation in C3(1)/ SV40 T/t-antigen transgenic mice. Cancer Res, 2001; 61: 7449-7455

42) Lubet RA, Gordon GB, Prough RA, Lei XD, You M, Wang Y, Grubbs CJ, Steele VE, Kelloff GJ, Thomas CF, Moon RD: Modulation of methylnitrosourea-induced breast cancer in Sprague Dawley rats by dehydroepiandrosterone: dose-dependent inhibition, effects of limited exposure, effects onperoxisomal enzymes, and lack of effects on levels of Ha-Ras mutations. Cancer Res, 1998; 58: 921 926

43) Perkins SN, Hursting SD, Haines DC, James SJ, Miller BJ, Phang JM: Chemoprevention of spontaneous tumorigenesis in nullizygous $\mathrm{p} 53$-deficient mice by dehydroepiandrosterone and its analog 16alpha-fluoro-5-androsten17-one. Carcinogenesis, 1997; 18: 989-994

44) Schwartz AG, Pashko LL: Cancer prevention with dehydroepiandrosterone and non-androgenic structural analogs. J Cell Biochem Suppl, 1995; 22: 210-217 